\title{
Cyclic helix B peptide ameliorates renal tubulointerstitial fibrosis induced by unilateral ureter obstruction via inhibiting NLRP3 pathway
}

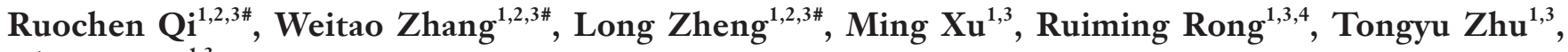 \\ Cheng Yang ${ }^{1,3}$
}

${ }^{1}$ Department of Urology, Zhongshan Hospital, Fudan University, Shanghai 200032, China; ${ }^{2}$ Shanghai Medical College, Fudan University, Shanghai 200032, China; '3 Shanghai Key Laboratory of Organ Transplantation, Shanghai 200032, China; ${ }^{4}$ Department of Transfusion, Zhongshan Hospital, Fudan University, Shanghai 200032, China

Contributions: (I) Conception and design: C Yang; (II) Administrative support: R Rong, T Zhu, C Yang; (III) Provision of study materials or patients: R Qi, L Zheng; (IV) Collection and assembly of data: W Zhang, L Zheng; (V) Data analysis and interpretation: R Qi, W Zhang, M Xu; (VI) Manuscript writing: All authors; (VII) Final approval of manuscript: All authors.

\#These authors contributed equally to this work.

Correspondence to: Dr. Cheng Yang. Department of Urology, Zhongshan Hospital, Fudan University, No. 180 Fenglin Road, Shanghai 200032, China. Email: esuperyc@163.com or yang.cheng1@zs-hospital.sh.cn.

Background: Renal fibrosis is the inevitable outcome of all progressive chronic kidney diseases (CKD) and leads to a gradual loss of renal function. We previously reported cyclic helix B peptide (CHBP), a novel synthesized peptide derived from erythropoietin, had shown effective renoprotection. In this study, we investigated the anti-fibrotic and renoprotective effect of CHBP in a murine renal tubulointerstitial fibrosis model induced by unilateral ureter obstruction (UUO).

Methods: Mice were subjected to the UUO model and CHBP was given intraperitoneally. To assess the therapeutic effects of CHBP, pathological injury, deposition of extracellular matrix (ECM) and the progression of epithelial-mesenchymal transition (EMT) were examined in vivo. The anti-fibrotic effects of CHBP was validated in vitro using TCMK-1 cells treated with TGF- $\beta 1$. Involvement of the NLRP3 pathway was demonstrated both in vivo and in vitro.

Results: CHBP significantly ameliorated renal tubulointerstitial injury and fibrosis in terms of ECM deposition. The EMT process was also alleviated after CHBP treatment. Similar therapeutic effects of CHBP were also observed in vitro in TGF- $\beta 1$ treated tubular epithelial cells (TECs). NLRP3/caspase-1/ IL-1 $\beta$ pathway was involved and activated upon injury, both in vivo and in vitro. While the activation of the NLRP3 pathway was found to be in negative correlation with CHBP treatment. CHBP could suppress the activation of NLRP3 and its downstream inflammatory mediators even with addition of extracellular ATP, a direct activator of the NLRP3 inflammasome.

Conclusions: Our results suggest that CHBP could effectively protect the kidney from renal tubulointerstitial fibrosis in the UUO model via counteracting the NLRP3/caspase-1/IL-1 $\beta$ pathway.

Keywords: Cyclic helix B peptide (CHBP); tubulointerstitial fibrosis; unilateral ureter obstruction (UUO); NLRP3; drug therapy

Submitted Nov 16, 2019. Accepted for publication Jan 10, 2020.

doi: $10.21037 / \mathrm{atm} .2020 .02 .12$

View this article at: http://dx.doi.org/10.21037/atm.2020.02.12 


\section{Introduction}

Chronic kidney diseases (CKD) have imposed great burden on public health worldwide. CKD alone accounted for $1.5 \%$ of deaths globally in 2012 according to WHO estimation (1). Renal fibrosis, especially tubulointerstitial fibrosis, characterized with deposition of extracellular matrix (ECM) within the kidney, occurs inevitably in all progressive CKD patients (2). Thus, it is of great importance to illustrate the intrinsic mechanism of how CKD progresses to renal fibrosis. Abundant studies have revealed the roles of different cell groups in the development of tubulointerstitial fibrosis, including renal tubular cells, endothelial cells as well as various innate and adaptive immune cells (3). Efforts have also been made to alleviate tubulointerstitial fibrosis targeting different cells and pathways related to it (4). However, effective clinical intervention to ameliorate or even reverse the progression of renal tubulointerstitial fibrosis is still lacking. Hence, it is of great urgency to explore a feasible treatment to deal with this challenge.

According to our previous work, a novel synthesized cyclic helix B peptide (CHBP) have shown great tissue protective role in different disease models (5-7). CHBP was a 11-amino acid peptide derived from erythropoietin (EPO). Its thioether-cyclized structure yielded a resistance to proteolytic degradation while performing a stronger tissue protective effect. The efficacy was achieved by a strong binding capacity to the tissue protective receptor (TPR). CHBP also eliminated the side-effects and toxicity of EPO by abandoning its erythropoietic function (8). Our previous work has shown that $\mathrm{CHBP}$ can effectively restore kidney function and ameliorate pathological changes in renal ischemia-reperfusion injury (IRI) model, aristolochic acid induced acute kidney injury (AKI) model and acute kidney graft rejection models (9-11). The tissue protective role of CHBP was not limited to the kidney but also was shown effective in an acute myocardial infarction model (7). However, up till now, there was only one research focused on the effect of CHBP on the progression from AKI to CKD following renal IRI and yielded positive results (6). Thus, we hypothesize that CHBP might also play a renoprotective role in the development of renal tubulointerstitial fibrosis.

The NLR family, pyrin domain-containing 3 (NLRP3) inflammasome is an intracellular complex that activates caspase-1, leading to the maturation of the proinflammatory cytokines IL-1 $\beta$ and IL-18. Studies regarding the NLRP3 pathway have surged during the past decade. Several studies have shed light on the involvement of the NLRP3 pathway in renal tubulointerstitial fibrosis (12-14). One research showed that NLRP3 deletion protected mice underwent 5/6 nephrectomy and alleviate renal fibrosis via alleviating mitochondrial abnormality (12). Another research applied unilateral ureter obstruction (UUO) model and found that NLRP3 deficiency attenuated renal fibrosis (13). These results led to the belief that activation of the NLRP3 pathway plays a detrimental role in the development and progression of tubulointerstitial fibrosis.

Hence, in the present study, we explored the renoprotective, especially the anti-fibrotic potential of CHBP in the mouse renal tubulointerstitial fibrosis model induced by UUO as well as in mouse tubular epithelial cells (TECs) in vitro. We also investigated whether the NLRP3 pathway is involved in the explicit molecular mechanism of CHBP-mediated renoprotection effect.

\section{Methods}

\section{Murine UUO model}

All experiments involving animal ethics were performed in accordance to the Guidelines of the Care and Use of Laboratory Animals of the Laboratory Animal Ethical Commission of Fudan University. All practices were performed with the approval of Animal Ethical Committee of Zhongshan Hospital, Fudan University. Male C57BL/6 mice weighing 20-25 g were purchased from Shanghai Slac Lab Animal, Co. Ltd. and accommodated in a SPFgrade animal room. The UUO model was established as we previously reported (12). In brief, Anesthesia was performed with $0.1 \mathrm{~g} / \mathrm{kg}$ pentobarbital intraperitoneally. Core body temperature was maintained at $37^{\circ} \mathrm{C}$ with a heating pad during the whole procedure. Midline incision was performed to fully expose the left kidney. The left ureter was carefully isolated then ligated with double 4-0 silk thread. The left ureter was then cut between the two ligations. In the sham operation group, the left ureter was only isolated but not ligated. $1 \mathrm{ml}$ of sterile saline was given intraperitoneally before the incision was sutured.

After analepsia, mice were divided randomly into 4 different groups ( $\mathrm{n}=6)$ according to different treatments: (I) Sham operation group; (II) UUO group; (III) UUO + low dose CHBP group (CHBP-L); (IV) UUO + high dose CHBP group (CHBP-H). CHBP was dissolved in sterile $\mathrm{PBS}$ and given intraperitoneally only once after the operation at a concentration of $8 \mathrm{nmol} / \mathrm{kg}$ for the low dose 
and $16 \mathrm{nmol} / \mathrm{kg}$ for the high dose CHBP group. The UUO group was given the same amount of sterile PBS in the same way. Animals were ethically sacrificed 2 weeks after operation.

\section{Cell culture and treatment}

TCMK-1 cell was purchased from the American Type Culture Collection (ATCC). Cells were cultured in DMEM/F12 with addition of $10 \%$ FBS. All cells were kept in a humidified incubator with $5 \% \mathrm{CO}_{2} / 95 \%$ air at $37{ }^{\circ} \mathrm{C}$. 24 hours before treatment, cells were seeded into 6-well plates (at a concentration of $1 \times 10^{5}$ cells/well). Cells were divided into 4 groups with different treatments in the first part of the in vitro experiment,: (I) control; (II) TGF- $\beta 1$; (III) TGF- $\beta 1+$ low dose CHBP (CHBP-L); (IV) TGF- $\beta 1$ + high dose CHBP (CHBP-H). TGF- $\beta 1$ was given at a concentration of $5 \mathrm{ng} / \mathrm{mL}$ in each group except the control. Low dose CHBP group was given an additional $5 \mu \mathrm{M}$ of CHBP and high dose CHBP group was given $10 \mu \mathrm{M}$ of CHBP besides TGF- $\beta 1$. Same amount of sterile PBS was given in the control group. In the second part of the in vitro experiment. Cells were divided into 7 groups: (I) control; (II) TGF- $\beta 1$; (III) high dose CHBP; (IV) ATP; (V) TGF- $\beta 1$ + high dose CHBP; (VI) TGF- $\beta 1$ + ATP; (VII) TGF- $\beta 1$ + high dose CHBP + ATP. TGF- $\beta 1$ was given at $5 \mathrm{ng} / \mathrm{mL}$, high dose CHBP was given at $10 \mu \mathrm{M}$ and ATP was given at $5 \mathrm{mM}$. All cells were further cultured for 72 hours before being harvested and processed for Western blot analysis.

\section{Pathological assessment}

Hematoxylin and eosin (H\&E) staining, Masson trichrome as well as Sirius red staining were applied in kidney sections (thickness $5 \mu \mathrm{m}$ ) to examine the pathological changes. All staining was performed as previously described (15). Semi-quantitative assessment of histological injury of $\mathrm{H} \& \mathrm{E}$ staining was performed by two independent renal pathologists according to the grading system described previously (6). Semi-quantitative assessment of Masson trichrome and Sirius red was performed with ImageJ 1.52a to measure the deposition of ECM in the renal interstitium.

\section{Immunobistochemistry and immunofluorescence staining}

Immunohistochemistry staining was performed in kidney sections (thickness $5 \mu \mathrm{m}$ ) with anti-fibronectin antibody (1:500, Abcam, Cambridge, UK) as we previously described (16). Immunofluorescence staining was applied in kidney sections (thickness $5 \mu \mathrm{m}$ ) and cultured cells according to our previous report (6). For the in vitro part, cells harvested after TGF- $\beta 1$ and CHBP treatment were first fixed with $4 \%$ paraformaldehyde for 15 mins and proceeded immunofluorescence staining with anti- $\alpha$-SMA (1:500, Abcam) and anti-E-cadherin antibodies (1:100, Cell Signaling Technology, Inc., Danvers, MA, USA) according to the protocol previously described (16).

\section{Terminal deoxynucleotidyl transferase dUTP nick end labeling (TUNEL) assay}

To examine the extent of apoptosis of TECs in kidney sections, TUNEL assay was applied using the in situ cell death detection kit (Roche, Basel, Switzerland) and processed according to the protocol provided by the manufacturer.

\section{Western blot analysis}

Protein was extracted from slice of kidney cortex and cultured cells and proceeded western blot analysis according to our previous report (7). In brief, protein samples (15 $\mu \mathrm{g}$ each) underwent electrophoresis and then were transferred on a PVDF membrane. The membrane was blocked with $1 \%$ BSA for 90 mins and then incubated with antibodies overnight at $4{ }^{\circ} \mathrm{C}$. Antibodies applied include anti-Collagen IV, anti-fibronectin, anti- $\alpha$-SMA, anti-caspase-1 $(1: 1,000$, Abcam), anti-COL1A1, anti-E-cadherin, anti-NLRP3, and anti-IL-1 $\beta$ antibodies (1:1,000, Cell Signaling Technology, Inc., Danvers, MA, USA).

\section{Statistical analysis}

All data were presented as mean \pm standard deviation $(\mathrm{SD})$. Statistical analysis was performed using Graphpad Prism 8 (GraphPad Software Inc., San Diego, CA, USA). Two-tailed independent Student's $t$-test was applied for comparison between two groups and one-way ANOVA for more groups. Statistical significance was considered as $\mathrm{P}<0.05$.

\section{Results}

\section{CHBP ameliorated pathological injury of the kidney}

To assess whether CHBP ameliorated renal tubulointerstitial fibrosis induced by UUO model, we first applied H\&E 
A
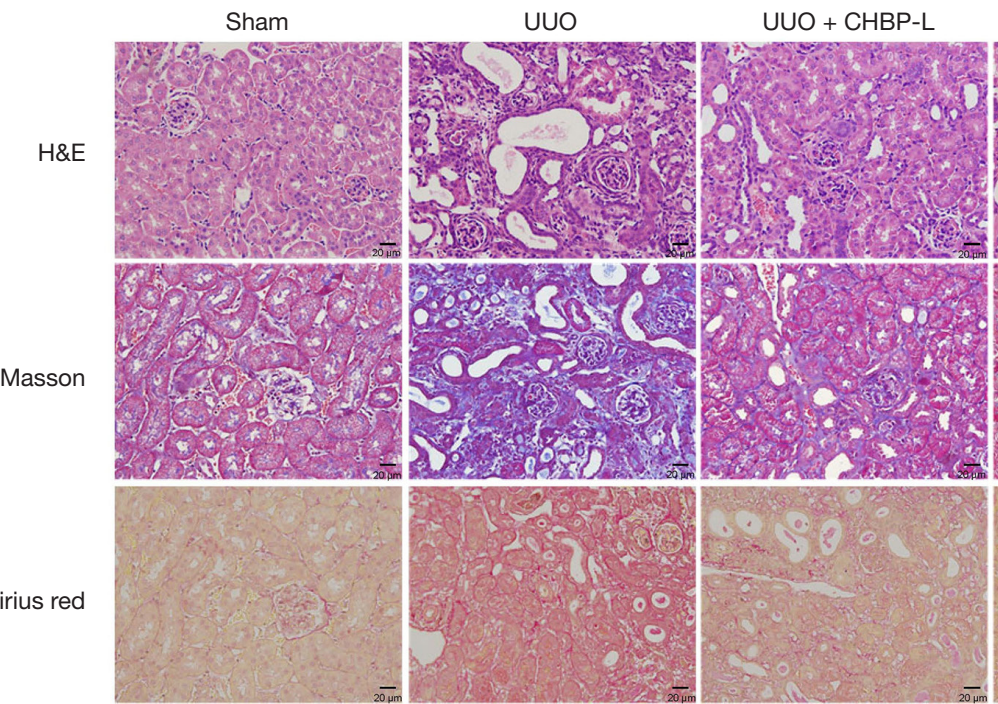

$\mathrm{UUO}+\mathrm{CHBP}-\mathrm{H}$

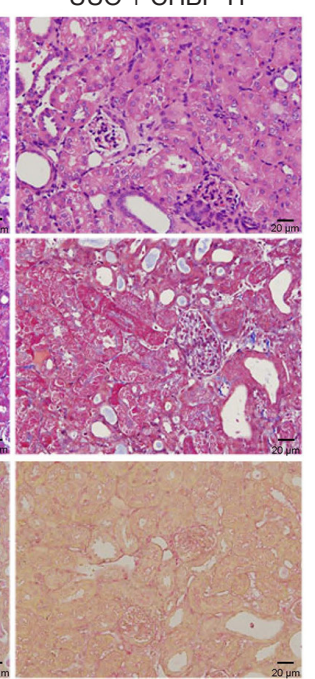

B
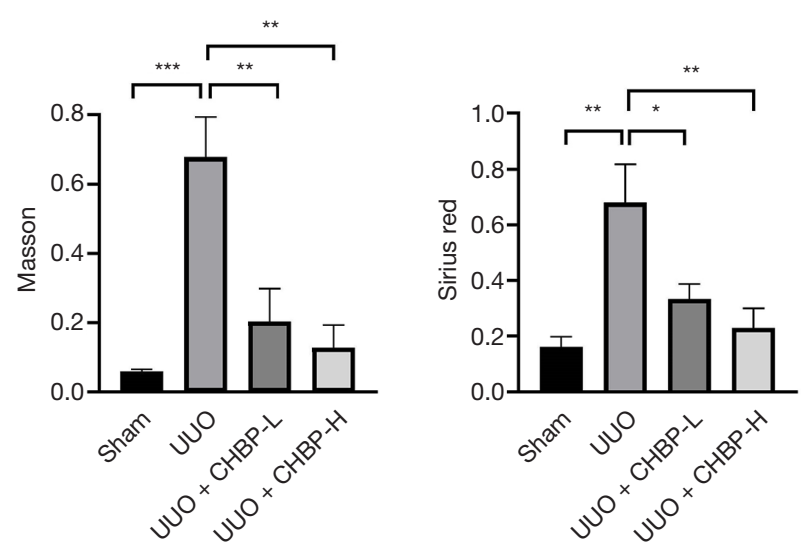

Figure 1 CHBP ameliorated pathological changes in UUO kidneys. (A) H\&E, Masson trichrome and Sirius red staining were performed on mice kidney sections 2 weeks post operation (200×); (B) semi-quantitative analysis for H\&E, Masson trichrome and Sirius red staining, respectively ( $\mathrm{n}=6) .{ }^{*}, \mathrm{P}<0.05$; $^{* *}, \mathrm{P}<0.01{ }^{* * *}, \mathrm{P}<0.001$. CHBP, cyclic helix B peptide; UUO, unilateral ureter obstruction.

staining to evaluate the histological injury of the kidney sections (Figure 1A), especially in the interstitial area and the tubular cells. UUO led to great dilation of the tubular lumen, loss of brush border, distortion of the interstitial structure as well as infiltration of inflammatory cells in the interstitial area compared with the sham operation group. These changes were partly offset with the introduction of low dose CHBP and further alleviated with high dose CHBP treatment. Tubular injury scores analysis also showed significant amelioration in CHBP treated groups (Figure 1B) .

Deposition of ECM, shown by Masson trichrome and Sirius red staining (Figure 1A), was also reduced in the CHBP treated groups compared with the untreated ones in a dose dependent manner. Semi-quantitative measurement of Masson and Sirius red staining also showed significant alleviation (Figure 1B). These results indicated that the pathological injuries as well as ECM deposition induced by UUO was relieved by CHBP treatment.

\section{CHBP alleviated renal tubulointerstitial fibrosis in the UUO model}

To further demonstrate the renoprotective effect of CHBP, we applied the immunohistochemistry staining of fibronectin, a representative ECM protein, in UUO kidney sections (Figure $2 A$ ) and processed western blot analysis to examine the expression of fibrosis associated proteins in each group (Figure 2B). Untreated UUO kidneys showed a larger amount of deposition of fibronectin in the interstitial 
A

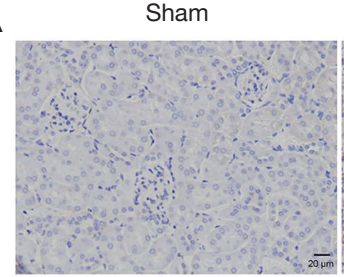

B

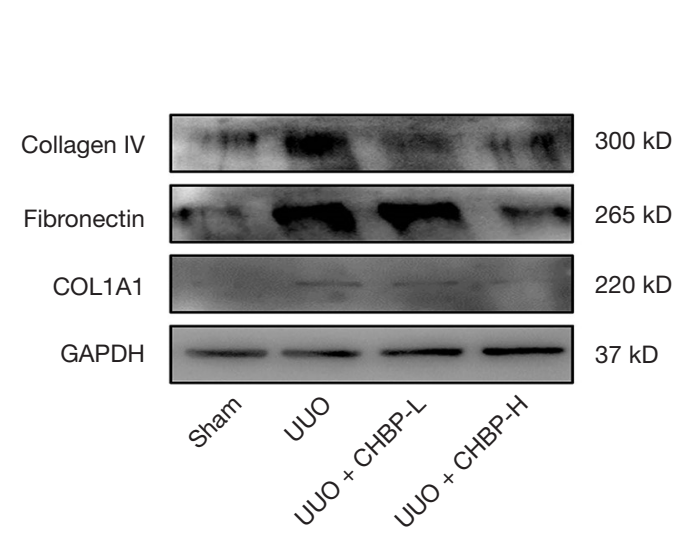

UUO

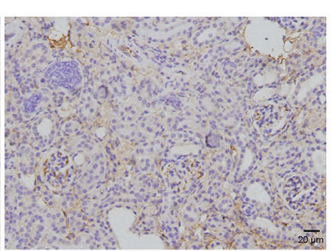

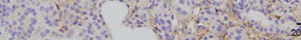

$\mathrm{UUO}+\mathrm{CHBP}-\mathrm{L}$

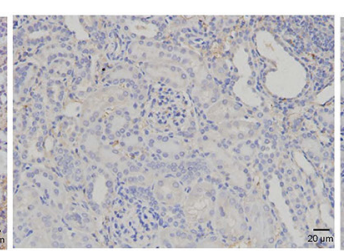

C

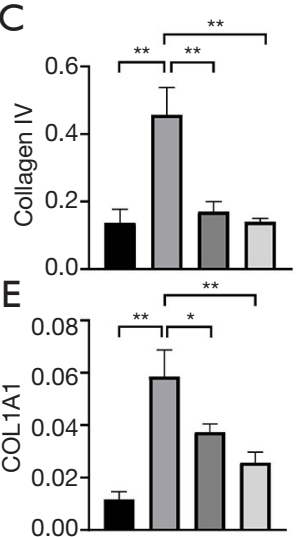

$\mathrm{UUO}+\mathrm{CHBP}-\mathrm{H}$
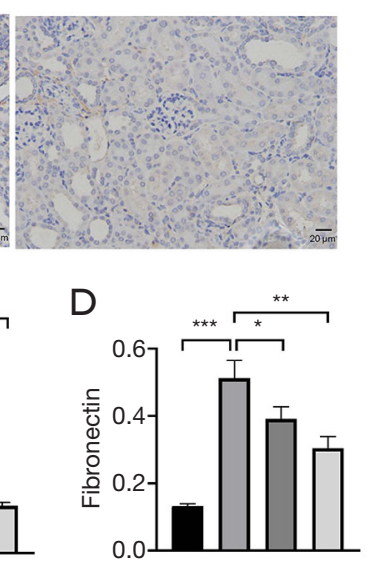

- Sham

口 UUO

口 UUO + CHBP-L

口 UUO + CHBP-H

Figure 2 CHBP alleviated renal tubulointerstitial fibrosis induced by UUO model. (A) Immunohistochemistry staining of fibronectin was performed on mice kidney sections 2 weeks after operation (200x); (B) expression of collagen IV, fibronectin and COL1A1 were determined by western blot analysis; quantitative analysis of (C) collagen IV, (D) fibronectin and (E) COL1A1 expression ( $\mathrm{n}=6)$. *, $\mathrm{P}<0.05$; **, $\mathrm{P}<0.01$; ***, $\mathrm{P}<0.001$. CHBP, cyclic helix B peptide; UUO, unilateral ureter obstruction.

area, suggesting that fibrosis was successfully induced in the UUO model. This phenomenon was ameliorated by low dose $\mathrm{CHBP}$ and further relieved in the high dose CHBP group. Western blot analysis also showed a decrease in the expression of Collagen IV, fibronectin and COL1A1 after CHBP treatment in a dose-dependent manner (Figure 2C,D,E). This indicates the progression of renal fibrosis was alleviated with CHBP treatment.

\section{CHBP ameliorated the EMT process as well as apoptosis in the UUO model}

Epithelial-mesenchymal transition (EMT), characterized with loss of epithelial features and acquisition of mesenchymal features in TECs, is an important source of activated myofibroblast in the renal interstitium and also a well-studied mechanism of the development of tubulointerstitial fibrosis (17). Thus, we hypothesized that inhibition of the EMT process may also play a part in the renoprotective role of CHBP in tubulointerstitial fibrosis. Therefore, we first applied immunofluorescence in the UUO kidneys sections (Figure 3A). We marked the most well recognized epithelial marker E-cadherin (green) as well as the mesenchymal marker $\alpha$-SMA (red) (18). Fluorescence showed that the epithelial phenotype of TECs in the sham operation group, characterized with high expression of E-cadherin and very little expression of $\alpha$-SMA, was turned to an opposite mesenchymal phenotype in the UUO group, indicating the progression of the EMT process along with renal fibrosis. The loss of E-cadherin was rescued in the CHBP treated group in a dose-dependent manner and meanwhile the expression of $\alpha$-SMA was reduced. Western blot analysis of E-cadherin as well as $\alpha$-SMA also showed similar results (Figure 3B,C,D). These suggests that treatment with CHBP also arrested the progression of EMT in the UUO-induced renal fibrosis model.

Our previous studies also showed that the tissue protective role of CHBP was partially achieved via inhibiting cell apoptosis (7). Thus, we also examine the extent of apoptosis with TUNEL assay (Figure $3 E, F$ ). Results showed obvious labelled fluorescence in the UUO group, while the fluorescence was reduced with CHBP treatment, suggesting that apoptosis was also inhibited with introduction of CHBP. 
A

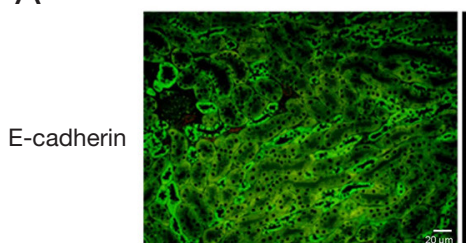

Sham
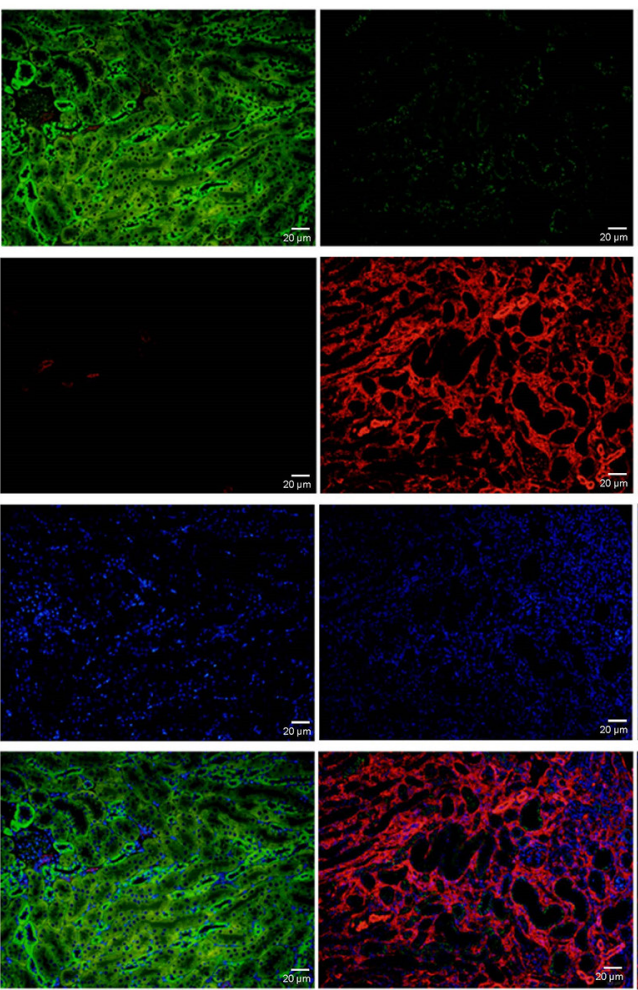

B

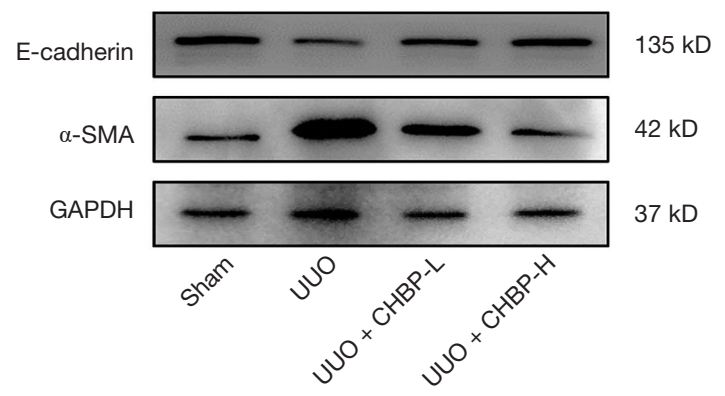

E

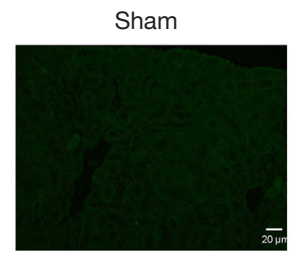

$\mathrm{UUO}+\mathrm{CHBP}-\mathrm{L}$

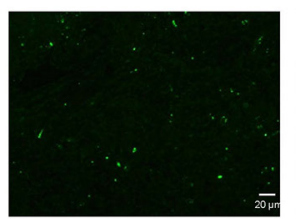

UบO

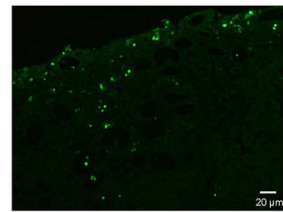

$\mathrm{UUO}+\mathrm{CHBP}-\mathrm{H}$

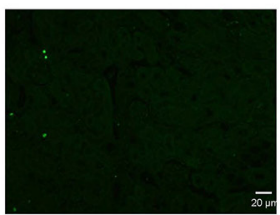

$\mathrm{UUO}+\mathrm{CHBP}-\mathrm{L}$
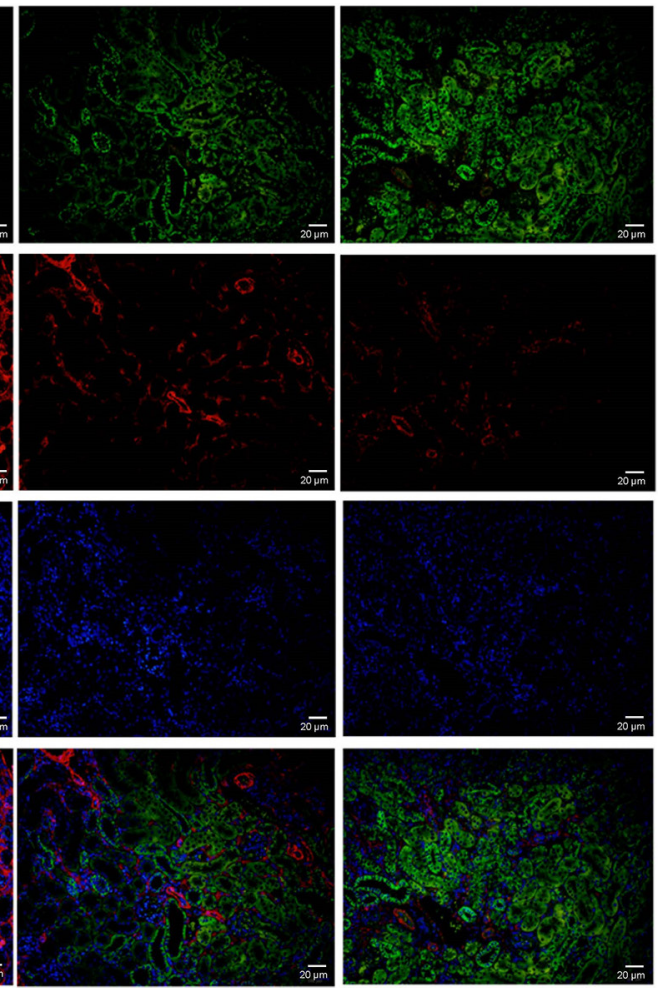

C

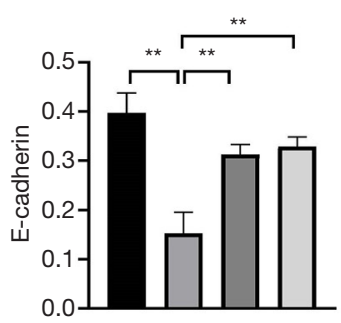

D
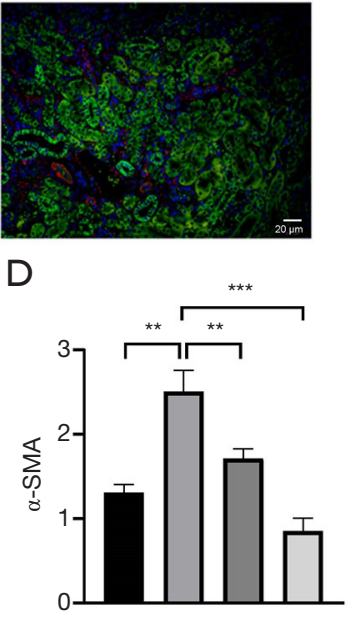

Sham

UUO

UUO + CHBP-L

$\mathrm{UUO}+\mathrm{CHBP}-\mathrm{H}$

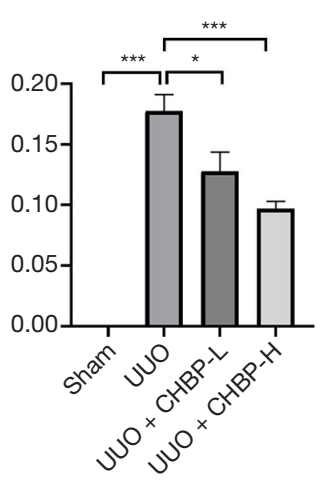

Figure 3 CHBP ameliorated the EMT process as well as apoptosis in vivo. (A) Immunofluorescence of E-cadherin and $\alpha$-SMA was performed on mice kidney sections 2 weeks after model establishment $(200 \times, n=6)$; (B) representative immunoblots of E-cadherin and $\alpha$-SMA and (C,D) the quantitative analysis, respectively; (E) fluorescence of TUNEL analysis in kidney sections and its (F) semi-quantitative analysis. * $\mathrm{P}<0.05$; **, $\mathrm{P}<0.01$; ${ }^{* * *}, \mathrm{P}<0.001$. CHBP, cyclic helix B peptide; EMT, epithelial-mesenchymal transition. 


\section{CHBP ameliorated ECM deposition as well as the EMT process in TECs in vitro}

To further verify the anti-fibrotic role of CHBP, we conducted in vitro experiment using mouse TEC line TCMK-1. TGF- $\beta 1$ pathway is a well-recognized profibrotic pathway and has been widely used to induce EMT and the pro-fibrotic phenotype in TECs $(12,19)$. After treatment and harvesting the cells, we conducted immunofluorescence staining to detect the expression of E-cadherin as well as $\alpha$-SMA (Figure $4 A$ ). Corresponding to the results of the in vivo experiments, fluorescence of E-cadherin (labelled with green) was rescued and $\alpha$-SMA (labelled with red fluorescence) was reduced after introduction of CHBP, indicating that CHBP could counteract the progression of EMT in vitro. Western blot analysis also showed a decreased expression of ECM proteins and changes in EMT markers after CHBP treatment (Figure 4B,C,D,E,F,G).

\section{CHBP inbibited the activation of NLRP3 pathway both in vivo and in vitro}

To verify whether the NLRP3/caspase-1/IL-1 $\beta$ pathway is involved in the anti-fibrotic molecular mechanism of CHBP, we conducted western blot analysis using kidney tissue and cell lysate (Figure $5 A, B$ ). We found that the NLRP3 inflammasome was activated in the UUO model, and its activation was downregulated in the CHBP treated group in a dose-depended manner. Cleaved caspase- 1 and matured IL- $1 \beta$, the downstream proteins of the NLRP3 inflammasome, also showed similar trend in different groups (Figure 5C,D,E,F,G,H). This suggest that the NLRP3 pathway is involved in the progression of renal fibrosis and is in negative correlation with CHBP treatment.

\section{CHBP attenuated renal tubulointerstitial fibrosis by inbibiting the NLRP3 patbway}

To further investigate the involvement of NLRP3 pathway, we activated the NLRP3 inflammasome using extracellular adenosine triphosphate (ATP). Introduction of ATP successfully upregulated NLRP3 expression as well as cleaved caspase- 1 and matured IL- $1 \beta$ in mouse TECs (Figure 6A). Meanwhile, increased expression of fibronectin and $\alpha$-SMA were also detected in response to extracellular ATP. Combined introduction of TGF- $\beta 1$ and ATP aggravated this phenomenon. In accordance with our previous results, treatment of CHBP downregulated the expression of fibrosis-associated proteins (Figure $6 B, C, D$ ) as well as the NLRP3 inflammasome activation in TGF- $\beta 1$ treated group (Figure 6E,F,G). Similar therapeutic effect was also observed in the TGF- $\beta 1+\mathrm{ATP}+\mathrm{CHBP}$ group. These results confirmed that the anti-fibrotic effect of CHBP was achieved by inhibiting NLRP3 inflammasome activation in TECs.

\section{Discussion}

Renal fibrosis, especially fibrosis in the interstitial area of the kidney, leads to progressive loss of residual renal function during the progression of CKD. Chronic allograft nephropathy in kidney transplant patients, now featured as interstitial fibrosis/tubular atrophy, also contributes to the eventual graft loss. Numerous studies have devoted to exploring the mechanisms of renal tubulointerstitial fibrosis and have made some advances. However, effective therapy to improve or rescue kidney function in patients with tubulointerstitial fibrosis is still absent in the clinical setting today. In the present study, we demonstrated for the first time that the EPO derived CHBP could ameliorate tubulointerstitial fibrosis induced by UUO model in vivo as well as the EMT progress in mouse TECs treated with TGF- $\beta 1$ in vitro. We further elucidated that the anti-fibrotic role of CHBP was achieved by inhibiting the activation of the NLRP3/caspase-1/IL-1 $\beta$ pathway.

Renal tubulointerstitial fibrosis could be initiated by a variety of injuries and this process involves a wide range of cells including macrophages, TECs, endothelial cells and fibroblasts. The progression of renal fibrosis is characterized with the deposition of ECM, for example, fibronectin and collagen fibers, in the interstitial area (20). In this study, Masson and Sirius red staining showed increased deposition of ECM in the UUO-induced tubulointerstitial fibrosis model. This phenomenon was attenuated in the CHBP treated mice group. Western blot analysis also showed decreased expression of fibronectin and collagen fibers after introduction of CHBP both in vivo and in vitro. These results demonstrated that CHBP could effectively ameliorate the deposition of ECM thus counteract the progression of tubulointerstitial fibrosis.

EMT, pictured as the transition from epithelial cells to mesenchymal cells, has been deemed as an important source of activated myofibroblasts in the injured kidney and therefore an important contributor to the progression of renal fibrosis (21). These TECs acquiring mesenchymal 
A
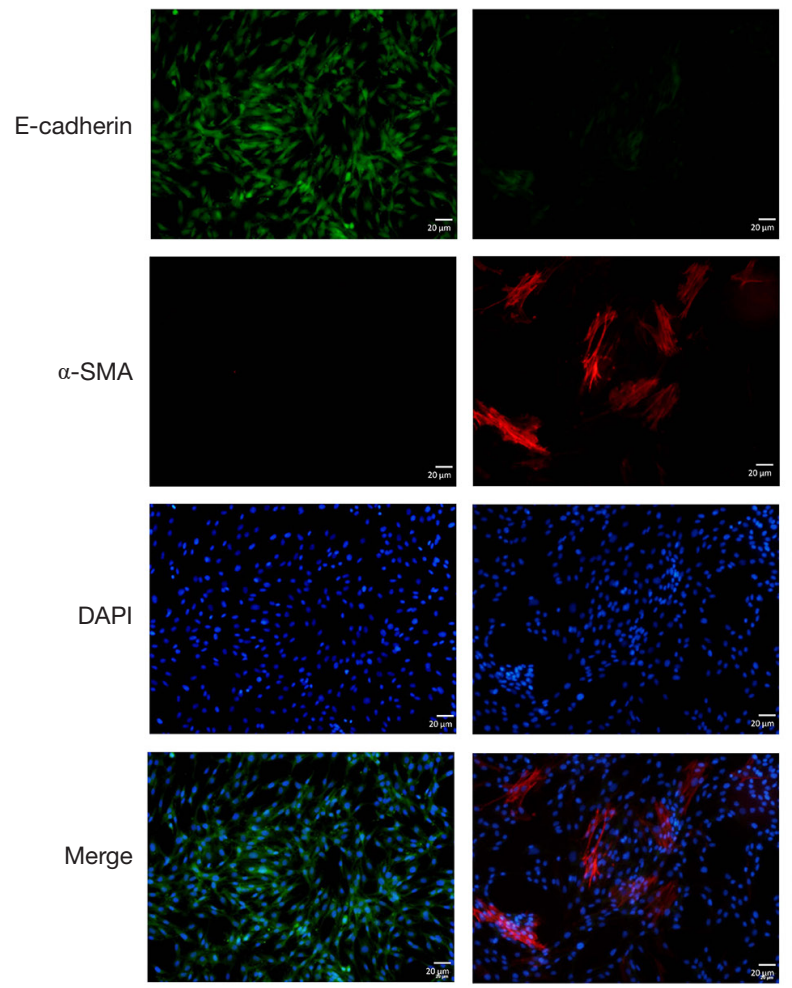

B

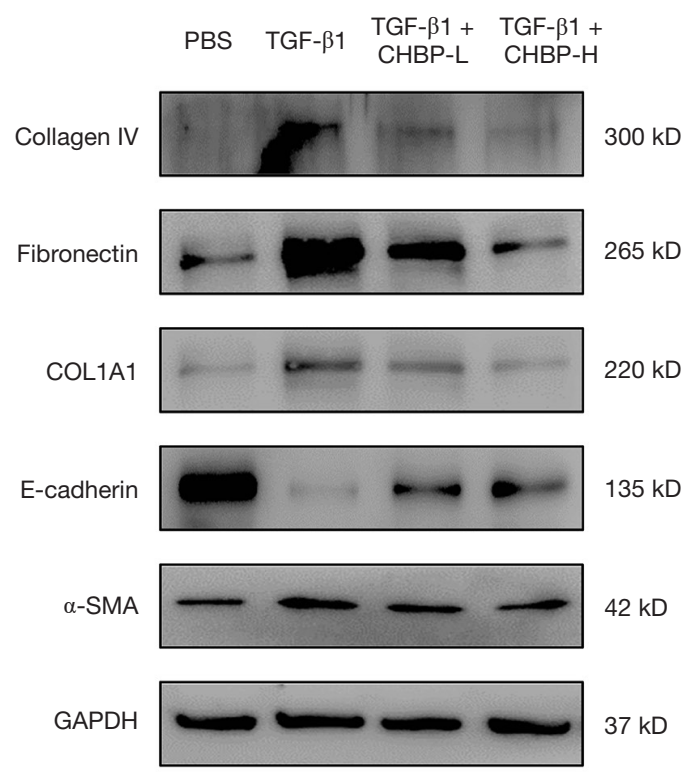

TGF- $\beta 1+$ CHBP-L
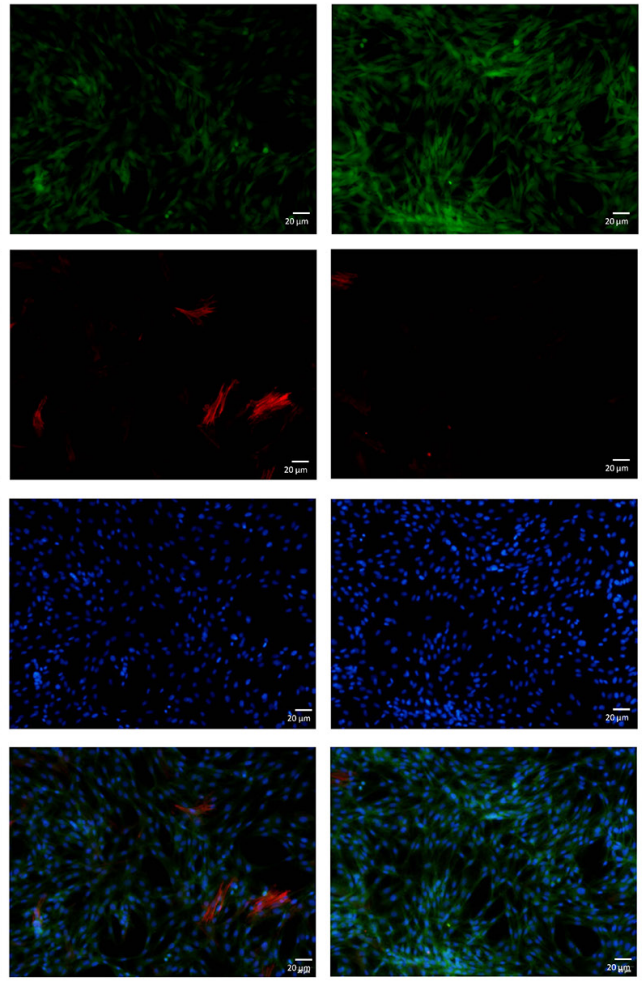

C

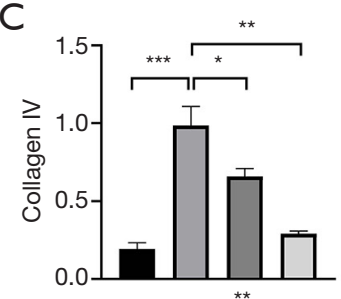

E
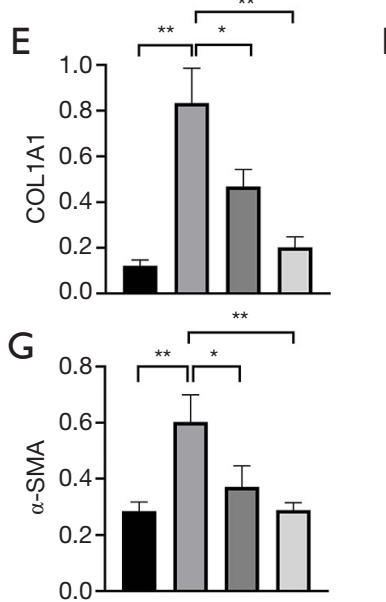
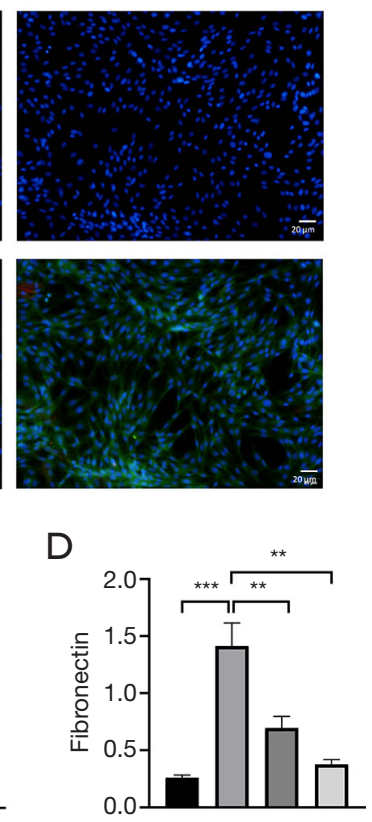

F

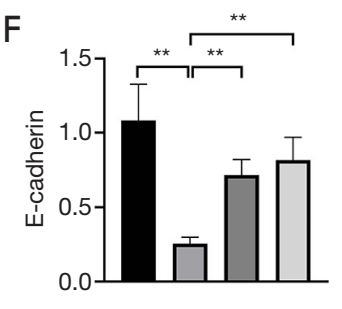

TGF- $\beta 1+$ CHBP-H
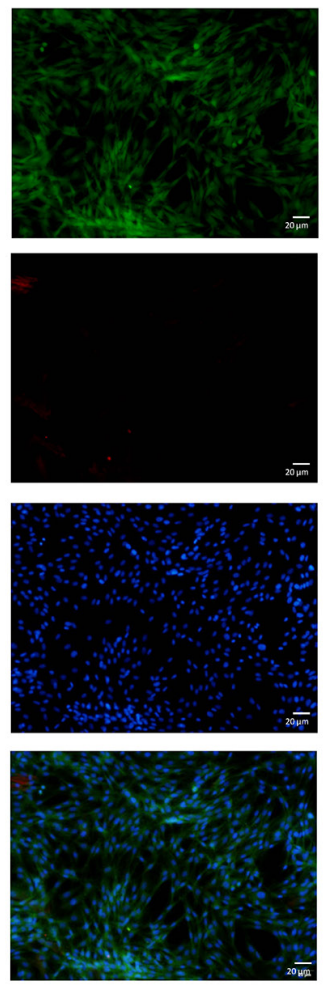

D

DBS

TGF- $\beta 1$

- TGF- $\beta 1+$ CHBP-L

TGF- $\beta 1+$ CHBP-H

Figure $4 \mathrm{CHBP}$ ameliorated ECM deposition as well as EMT process in vitro induced by TGF- $\beta 1$. (A) Immunofluorescence of E-cadherin and $\alpha$-SMA in cultured TCMK-1 cells $(200 \times, n=3)$; (B) representative immunoblots of ECM proteins including collagen IV, fibronectin and COL1A1, as well as EMT markers, E-cadherin and $\alpha$-SMA, and their $(\mathrm{C}, \mathrm{D}, \mathrm{E}, \mathrm{F}, \mathrm{G})$ quantitative analysis, respectively. *, $\mathrm{P}<0.05 ;{ }^{* *}, \mathrm{P}<0.01$; ***, $\mathrm{P}<0.001$. CHBP, cyclic helix B peptide; EMT, epithelial-mesenchymal transition. 
A $\begin{array}{llll}\text { Sham UUO } & \text { UUO + } & \text { UUO + } \\ \text { CHBP-L } & \text { CHBP-H }\end{array}$ NLRP3

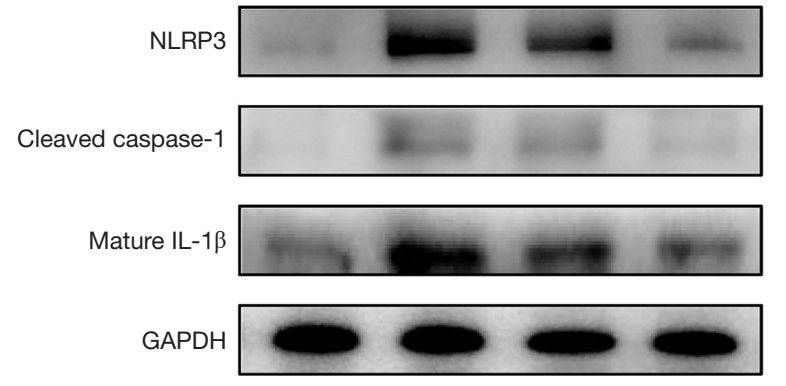
$-130 \mathrm{kD}-$ $\begin{array}{llll}\text { B PBS TGF- } \beta 1 & \begin{array}{c}\text { TGF- } \beta 1+ \\ \text { CHBP-L }\end{array} & \text { TGF- } \beta 1+ \\ \text { CHBP-H }\end{array}$

$-20 \mathrm{kD}-$
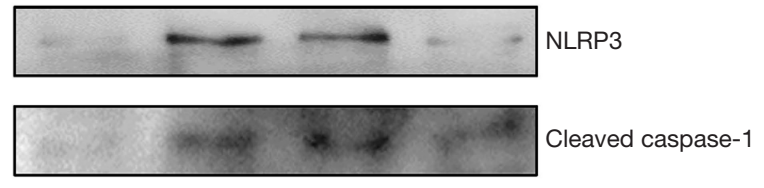

$-17 \mathrm{kD}-$

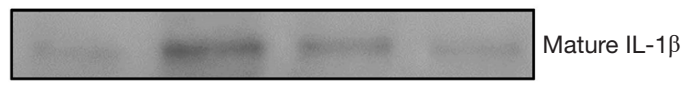

$-37 \mathrm{kD}-$

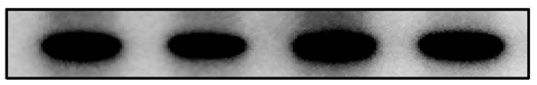

GAPDH

C

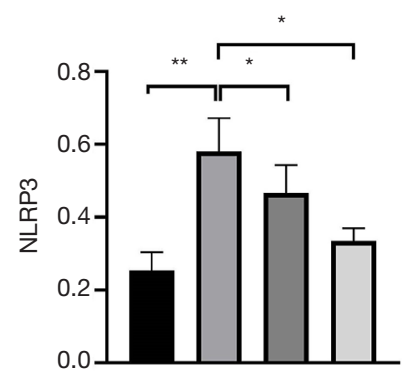

$\mathrm{F}$

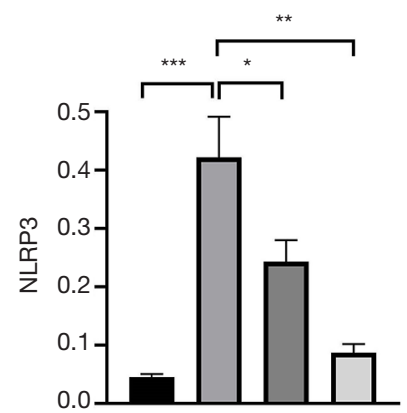

D

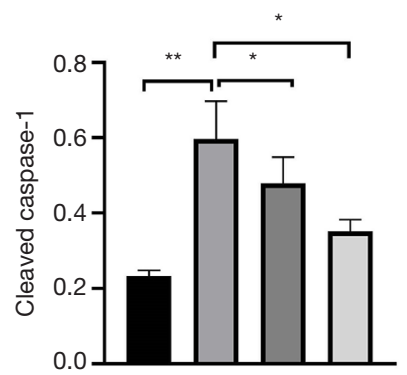

G

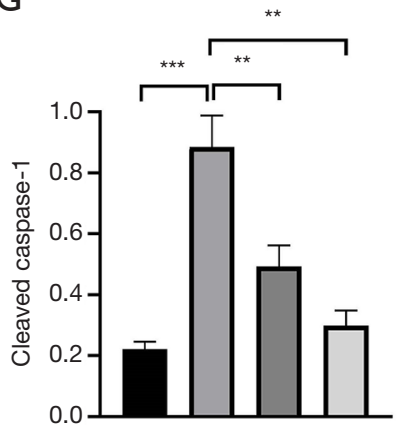

$E$

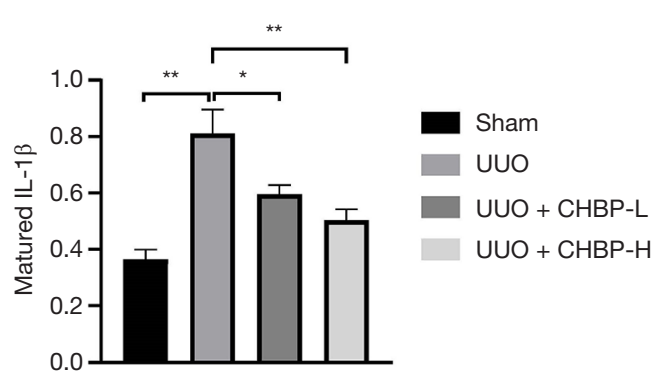

$\mathrm{H}$

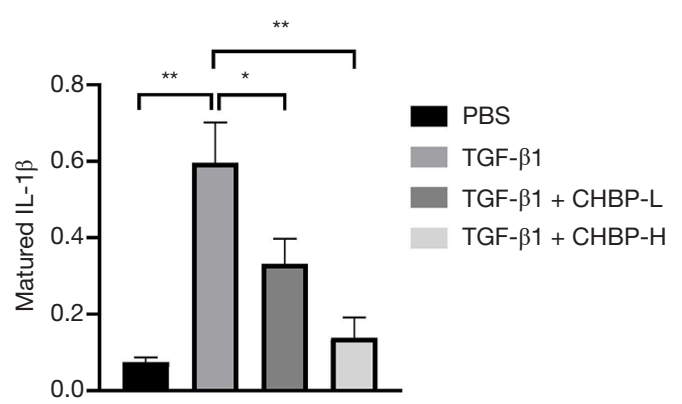

Figure 5 CHBP inhibited the activation of NLRP3 pathway both in vivo and in vitro. Representative immunoblots of NLRP3, cleaved caspase-1 and matured IL-1 $\beta$ (A) in vivo and (B) in vitro and their (C,D,E,F,G,H) quantitative analysis, respectively. *, $\mathrm{P}<0.05$; ${ }^{* *}, \mathrm{P}<0.01$; ***, $\mathrm{P}<0.001$. CHBP, cyclic helix B peptide.

phenotype can secrete a wide range of pro-inflammatory cytokines which further aggravate renal injury. Meanwhile, these cells also become a source of ECM thus directly push forward the progression of renal fibrosis (17). In the current study, we also observed the activation of EMT in the animal model as well as in cultured cells using immunofluorescence. This process was alleviated by treatment of CHBP, manifested with restoration of E-cadherin expression and reduced staining of $\alpha$-SMA. Our previous study has shown that CHBP could counteract apoptosis in TECs upon injury (22), this finding was also confirmed in the present study with TUNEL analysis. This demonstrate that the protective effect of CHBP in TECs is probably holistic, by targeting both the EMT process meanwhile counteracting apoptosis.

Sustained inflammation is an important mediator of renal tubulointerstitial fibrosis. Among a variety of proinflammatory cytokines, IL- $1 \beta$ plays a key role. The activation of the NLRP3 pathway by danger-associated molecular patterns (DAMPs) and pathogen-associated 
A

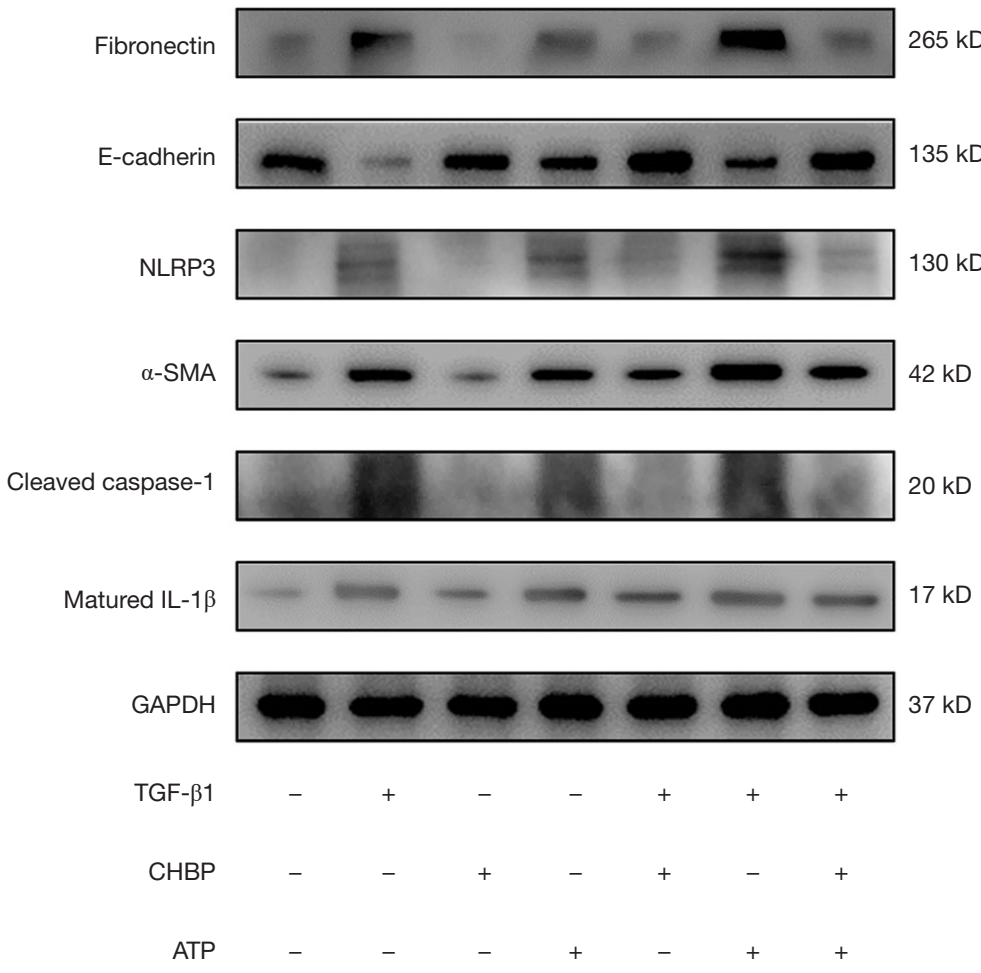

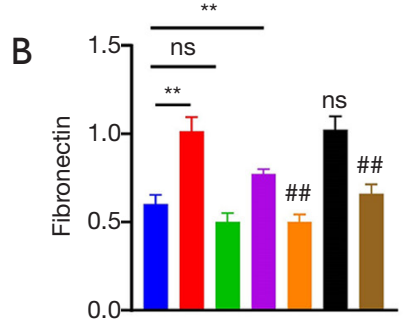

C

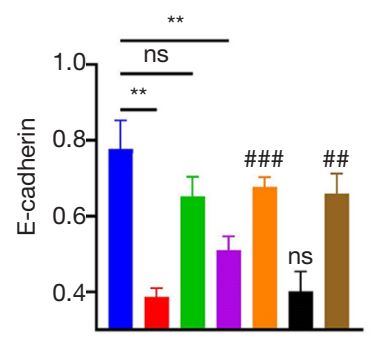

D

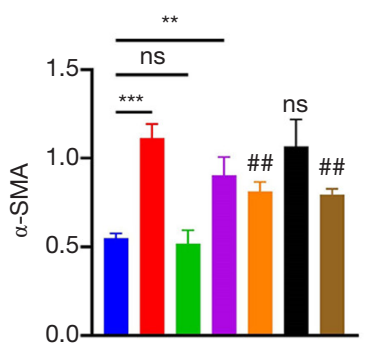

E

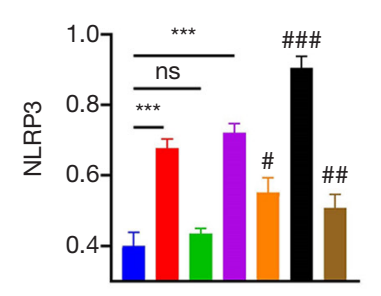

F

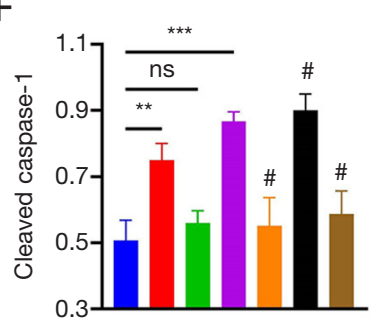

G

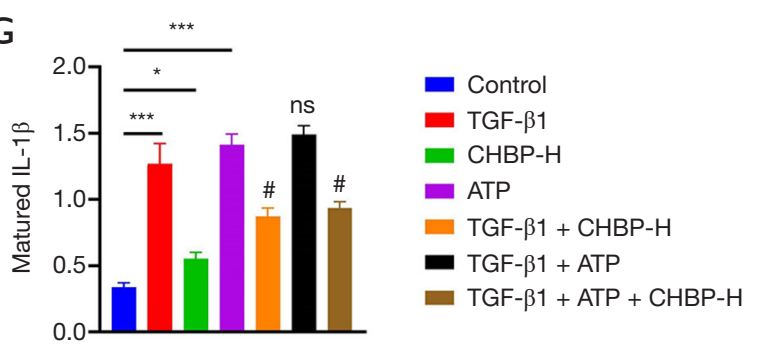

Figure 6 CHBP ameliorated renal tubulointerstitial fibrosis by inhibiting NLRP3 pathway. Cultured TCMK-1 cells were treated with TGF- $\beta 1$, ATP and CHBP in different groups. (A) Representative immunoblots of fibronectin, E-cadherin, NLRP3, $\alpha$-SMA, cleaved caspase-1 and matured IL-1 $\beta$; (B,C,D,E,F,G) their quantitative analyses were also shown, respectively. *, $\mathrm{P}<0.05 ;{ }^{* *}, \mathrm{P}<0.01 ;{ }^{* * *}, \mathrm{P}<0.001$

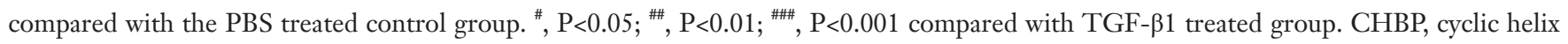
B peptide.

molecular patterns (PAMPs)is an important intracellular pathway which contributes to the final maturation of IL-1 $\beta$ (23,24). Several recent studies have reviewed the activation of NLRP3 pathway in CKD and renal fibrosis $(12,13,19)$. Depletion of NLRP3 in vivo attenuated renal fibrosis in mice 5/6 nephrectomy model, which suggested that the NLRP3 inflammasome activation took part in the progression of renal fibrosis. In the current study, we illustrated that intervention of CHBP downregulated the expression of NLRP3 as well its downstream mediators, cleaved caspase- 1 and matured IL-1 $\beta$ (Figure 7). This finding was confirmed in UUO animal model as well as the TGF- $\beta 1$ treated TECs. To further investigate the mechanism, we introduced ATP in TECs cell culture. Numbers of studies have shown that extracellular ATP acts as an activator of the NLRP3 pathway $(25,26)$. 


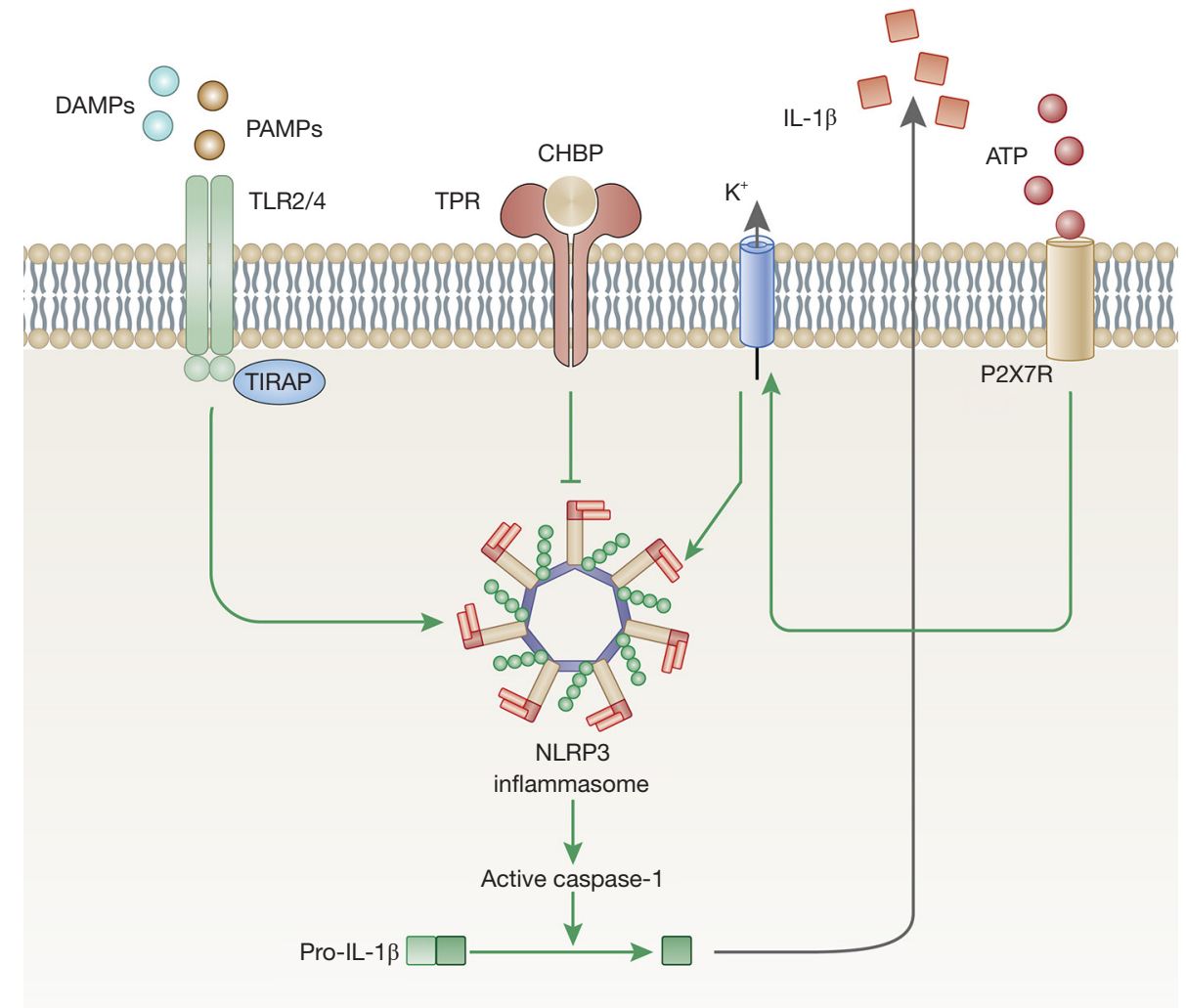

Figure 7 Schematic view of the anti-fibrotic mechanism of CHBP. Upon injuries, the Toll-like receptors (TLRs) on tubular epithelial cell surface recognize a variety of signals, including damage-associated molecular patterns (DAMPs) and pathogen-associated molecular patterns (PAMPs). This triggers the oligomerization of the NLRP3 inflammasome, which proceeds the activation of caspase-1, and further, the generation of inflammatory cytokines, IL-1 $\beta$. Injuries also lead to release of extracellular ATP, which binds to the P2X7R on cell surface and causes an efflux of K+. This further activates the NLRP3 inflammasome and echoes the inflammation process. CHBP could effectively counteract the activation of the NLRP3 pathway thus protect tubular cells from excessive inflammation. CHBP, cyclic helix B peptide.

Binding of ATP to the ATP-gated cation channel P2X7R causes an efflux of $\mathrm{K}^{+}$, which further activates the NLRP3 inflammasome (25). In this study, we found that CHBP could still reduce the expression of fibrosis-associated proteins as well as downregulate the NLRP3 pathway activation even with addition of extracellular ATP, which further confirmed the inhibition of NLRP3 pathway by CHBP. We hypothesize that the disturbance of maturation of IL- $1 \beta$ by CHBP leads to a constraint in the inflammation within the kidney and therefore attenuate kidney injury as well as renal fibrosis.

However, there are still some limitations in this study. We focused on tubulointerstitial fibrosis and applied mice UUO model in the in vivo experiment to examine the antifibrotic effect of CHBP. Though UUO is a well-established animal model for renal tubulointerstitial fibrosis, its clinical significance is minor. Compared to the progressive kidney failure and renal fibrosis caused by hypertension and diabetes, obstructive nephropathy induced tubulointerstitial fibrosis, for instance renal calculus and urological deformity, is less commonly seen in the clinical settings. Thus, we only demonstrated the anti-fibrotic role of CHBP in obstructive induced tubulointerstitial fibrosis in this study, whether this EPO-derived peptide has similar therapeutic effect in hypertensive or diabetic kidney diseases remains to be explored.

\section{Conclusions}

In conclusion, we demonstrated for the first time that the EPO derived cyclic peptide CHBP has a renoprotective effect in UUO-induced renal tubulointerstitial fibrosis mice model. Treatment of CHBP successfully alleviated the pathological changes, ECM deposition, cell apoptosis 
as well as the EMT process during the progression of tubulointerstitial fibrosis. Further exploration showed that the protective effect of CHBP was achieved by inhibiting the activation of NLRP3 inflammasome as well as its downstream factors, cleaved caspase- 1 and matured IL$1 \beta$. This study further confirmed the efficacy of CHBP in ameliorating renal fibrosis and shed some new insight into the molecular mechanism within it. Therefore, this study suggests that CHBP be a potential drug for renal tubulointerstitial fibrosis in the future.

\section{Acknowledgments}

Funding: This study was supported by the National Key R\&D Program of China (2018YFA0107502 to C Yang, 2018YFA0107501 to R Rong), National Natural Science Foundation of China (81770746 to C Yang, 81770747 to R Rong), Shanghai Rising-Star Program (19QA1406300 to C Yang), the Medical and Health Talents Training Plan for the Excellent Youth of Shanghai Municipal (2018YQ50 to C Yang) and the Science and Technology Commission of Shanghai Municipality (16431902300 to T Zhu).

\section{Footnote}

Conflicts of Interest: The authors have no conflicts of interest to declare.

Ethical Statement: The authors are accountable for all aspects of the work in ensuring that questions related to the accuracy or integrity of any part of the work are appropriately investigated and resolved. All practices were performed with the approval of Animal Ethical Committee of Zhongshan Hospital, Fudan University (No. Y2018-249).

Open Access Statement: This is an Open Access article distributed in accordance with the Creative Commons Attribution-NonCommercial-NoDerivs 4.0 International License (CC BY-NC-ND 4.0), which permits the noncommercial replication and distribution of the article with the strict proviso that no changes or edits are made and the original work is properly cited (including links to both the formal publication through the relevant DOI and the license). See: https://creativecommons.org/licenses/by-nc-nd/4.0/.

\section{References}

1. Webster AC, Nagler EV, Morton RL, et al. Chronic
Kidney Disease. Lancet 2017;389:1238-52.

2. Liu Y. Cellular and molecular mechanisms of renal fibrosis. Nat Rev Nephrol 2011;7:684-96.

3. Humphreys BD. Mechanisms of Renal Fibrosis. Annu Rev Physiol 2018;80:309-26.

4. Tampe D, Zeisberg M. Potential approaches to reverse or repair renal fibrosis. Nat Rev Nephrol 2014;10:226-37.

5. Li L, Lin M, Zhang L, et al. Cyclic helix B peptide protects HK2 cells from oxidative stress by inhibiting ER stress and activating Nrf2 signalling and autophagy. Mol Med Rep 2017;16:8055-61.

6. Yang C, Cao Y, Zhang Y, et al. Cyclic helix B peptide inhibits ischemia reperfusion-induced renal fibrosis via the PI3K/Akt/FoxO3a pathway. J Transl Med 2015;13:355.

7. Yang C, Zhang C, Jia J, et al. Cyclic helix B peptide ameliorates acute myocardial infarction in mice by inhibiting apoptosis and inflammatory responses. Cell Death Discov 2019;5:78.

8. Zhang C, Yang C, Zhu T. From Erythropoietin to Its Peptide Derivatives: Smaller but Stronger. Curr Protein Pept Sci 2017;18:1191-4.

9. Yang $\mathrm{C}, \mathrm{Xu} Z$, Zhao Z, et al. A novel proteolysisresistant cyclic helix B peptide ameliorates kidney ischemia reperfusion injury. Biochim Biophys Acta 2014;1842:2306-17.

10. Zeng Y, Zheng L, Yang Z, et al. Protective effects of cyclic helix B peptide on aristolochic acid induced acute kidney injury. Biomed Pharmacother 2017;94:1167-75.

11. Yang C, Zhang Y, Wang J, et al. A novel cyclic helix $\mathrm{B}$ peptide inhibits dendritic cell maturation during amelioration of acute kidney graft rejection through Jak-2/ STAT3/SOCS1. Cell Death Dis 2015;6:e1993.

12. Gong $\mathrm{W}, \mathrm{Mao} \mathrm{S}, \mathrm{Yu} \mathrm{J}$, et al. NLRP3 deletion protects against renal fibrosis and attenuates mitochondrial abnormality in mouse with 5/6 nephrectomy. Am J Physiol Renal Physiol 2016;310:F1081-8.

13. Guo H, Bi X, Zhou P, et al. NLRP3 Deficiency Attenuates Renal Fibrosis and Ameliorates Mitochondrial Dysfunction in a Mouse Unilateral Ureteral Obstruction Model of Chronic Kidney Disease. Mediators Inflamm 2017;2017:8316560.

14. Nam SA, Kim WY, Kim JW, et al. Autophagy attenuates tubulointerstital fibrosis through regulating transforming growth factor-beta and NLRP3 inflammasome signaling pathway. Cell Death Dis 2019;10:78.

15. Yang B, Jain S, Pawluczyk IZ, et al. Inflammation and caspase activation in long-term renal ischemia/reperfusion injury and immunosuppression in rats. Kidney Int 
2005;68:2050-67.

16. Zheng L, Li L, Qi G, et al. Transplantation of Telocytes Attenuates Unilateral Ureter Obstruction-Induced Renal Fibrosis in Rats. Cell Physiol Biochem 2018;46:2056-71.

17. Qi R, Yang C. Renal tubular epithelial cells: the neglected mediator of tubulointerstitial fibrosis after injury. Cell Death Dis 2018;9:1126.

18. Kriz W, Kaissling B, Le Hir M. Epithelial-mesenchymal transition (EMT) in kidney fibrosis: fact or fantasy? J Clin Invest 2011;121:468-74.

19. Wang S, Li Y, Fan J, et al. Interleukin-22 ameliorated renal injury and fibrosis in diabetic nephropathy through inhibition of NLRP3 inflammasome activation. Cell Death Dis 2017;8:e2937.

20. Meng XM, Nikolic-Paterson DJ, Lan HY. Inflammatory processes in renal fibrosis. Nat Rev Nephrol 2014;10:493-503.

21. Grande MT, Lopez-Novoa JM. Fibroblast activation and myofibroblast generation in obstructive nephropathy. Nat Rev Nephrol 2009;5:319-28.

22. Wang S, Zhang C, Niyazi S, et al. A novel cytoprotective

Cite this article as: Qi R, Zhang W, Zheng L, Xu M, Rong R, Zhu T, Yang C. Cyclic helix B peptide ameliorates renal tubulointerstitial fibrosis induced by unilateral ureter obstruction via inhibiting NLRP3 pathway. Ann Transl Med 2020;8(5):167. doi: 10.21037/atm.2020.02.12 peptide protects mesenchymal stem cells against mitochondrial dysfunction and apoptosis induced by starvation via Nrf2/Sirt3/FoxO3a pathway. J Transl Med 2017;15:33.

23. Swanson KV, Deng M, Ting JPY. The NLRP3 inflammasome: molecular activation and regulation to therapeutics. Nat Rev Immunol 2019;19:477-89.

24. Leu WJ, Chen JC, Guh JH. Extract From Plectranthus amboinicus Inhibit Maturation and Release of Interleukin 1beta Through Inhibition of NF-kappaB Nuclear Translocation and NLRP3 Inflammasome Activation. Front Pharmacol 2019;10:573.

25. Xie Q, Shen WW, Zhong J, et al. Lipopolysaccharide/ adenosine triphosphate induces IL1beta and IL18 secretion through the NLRP3 inflammasome in RAW264.7 murine macrophage cells. Int J Mol Med 2014;34:341-9.

26. Shi J, Zhao W, Ying H, et al. Estradiol inhibits NLRP3 inflammasome in fibroblast-like synoviocytes activated by lipopolysaccharide and adenosine triphosphate. Int J Rheum Dis 2018;21:2002-10. 